\title{
Robot-Assisted Pancreatic Surgery: A Structured Approach to Standardization of a Program and of the Operation
}

\author{
Charlotte Friederieke Müller-Debus Michael Thomaschewski \\ Markus Zimmermann Ulrich F. Wellner Dirk Bausch Tobias Keck \\ Department of Surgery, University Hospital of Schleswig Holstein (UKSH), Lübeck, Germany
}

\section{Keywords}

Pancreas - Whipple $\cdot$ Robot

\begin{abstract}
Introduction: The use of robots in minimal invasive surgery has become increasingly common in recent years. Robot-assisted pancreatoduodenectomy preponderates against a laparoscopic procedure especially due to the greater flexibility of instruments and therefore a better handling and a better angulation. Furthermore, there are benefits of enlarged 3-D visibility, software-based tremor control, and reduced physical exertion of the surgeon. Methods and Results: This review delivers a point-by-point approach to the setup of a robot-assisted pancreatic program and a detailed approach to robot-assisted pancreatoduodenectomy. Results: In our standardized standard operating procedure approach we use 5 trocars, i.e., 4 robotic trocars and 1 assist trocar. We prefer the position of the robot ports in a straight horizontal line with a distance of $20 \mathrm{~cm}$ from the operational field. The operation is dissected into 11 standardized procedural steps as follows: (1) access to the pancreas and visualization, (2) extended Kocher manoeuvre, (3) lower rim and mesentericoportal axis, (4) upper rim and hepatoduodenal ligament, (5) dissection of the pancreatic neck, (6) mesenteric root and pars IV duodeni, (7) mesopancreas, (8) pancreatic anastomosis reconstruction, (9) bilioenteric anastomosis, (10) dudenojejunal anastomosis, and (11) drainage and closure. The setup of the pancreas program and the structured approach to
\end{abstract}

complex pancreatic resections are elucidated. Summary: This review describes the approach to robot-assisted pancreatic surgery in a high-volume pancreas center on a structural and procedural level to support the establishment of such programs at other locations.

๑) 2020 S. Karger AG, Basel

\section{Introduction}

The use of robots in minimal invasive surgery has become increasingly common in recent years. Up to now the advantage of using robot-assisted surgery as compared to laparoscopic surgery could not be proven because of insufficient corresponding research and a lack of surveys. Especially in complex visceral surgery, however, with difficult reconstructive procedures such as pancreatic surgery, the wide spread implementation of minimal invasive surgery has not overcome technical hurdles and its implementation has not exceeded the level of a few dedicated centers. In pancreatic surgery we particularly see advantages in the greater flexibility of instruments and therefore a better handling especially during complex steps of an operation. These steps in pancreatic surgery comprise, not exclusively, preparation of the hepatoduodenal ligament, reconstruction of the bilioenteric passage by a biliodigestive anastomosis in particular of the notextended bile duct, reconstruction of the pancreatic anastomosis, and complex vascular resection and reconstruc- 
tion. Apart from the technical benefits that are based on angulating instruments, the surgeon benefits from enlarged 3-D visibility, software-based tremor control, and reduced physical exertion due to the ergonomic sitting position.

This article provides an in-depth description of the setup and systematic implementation of a pancreatic robotic program at our university hospital and focuses on standard operation procedures for robot-assisted pancreatic procedures. The program has been implemented in a high-volume pancreatic center ( $>100$ resections/year) and has now reached over 50 robot-assisted pancreatic resections.

\section{Indications for Robotic-Assisted Surgery}

Planning of an operation based on the use of a robot is preceded by fixed indication to a pancreatic resection, which can be cystic tumors, neuroendocrine tumors or tumors with malignancy. In the beginning of the implementation of the robot assisted operations we focussed on a previously recommended approach from an expert group on the implementation of laparoscopic pancreatic surgery [1]. The indication for carrying out a pancreatic operation with the robot followed the same criteria as for laparoscopic pancreatic surgery. In the beginning of the program malignancies with proximity of the tumor to the mesentericoportal axis were excluded from the indications. Patients with an extensive operation history, as well as those with severe cardiopulmonal comorbidity or an elevated BMI ( $>35)$, were excluded from the robot-assisted approach and were operated on via laparotomy. Patients with an insignificant operation history can often be operated on by a robot. In such cases we start with a laparoscopy first and perform an adhesiolysis if necessary.

\section{Setup of the Robotic Program}

A systematic setup of the pancreatic robotic program at the university hospital in Lübeck was pursued. Two high-volume pancreatic surgeons ( $>300$ pancreatic resections for each) underwent the console training provided by Intuitive Surgical (Intuitive, Sunnyvale, CA, USA) comprising robotic setup and functionality, evaluated simulator training $(>80 \mathrm{~h})$, and animal wet lab training. Four residents and 6 OR nurses were trained simultaneously as table assistants and certified Da Vinci nurses. The robot was set up in a remote $\mathrm{OR}$ area enabling team training and team approaches. At the beginning of the program, operations of a reduced complexity were performed by the 2 surgeons for safe implementation of robot-assisted surgery (cholecystectomy, fundoplication, resection of localized stomach tumors, and adrenalectomies). For use of the robot in pancreatic surgery, we followed the expert consensus for implementation of laparoscopic pancreatic surgery, meaning that left-sided resections, enucleations, and segmental resections were first applied. After 20 procedures, more complex resections of the pancreatic head were approached. Before this, the surgeons underwent additional biotissue training with performance evaluation and visited a high-volume robotic pancreatic program (Prof. Zeh and Zureikat, University of Pittsburg Medical Center, University of Pittsburgh) [2,3]. This was followed by a training session based on cadaver training for both surgeons under the supervision of a European expert surgeon in robot-assisted pancreatic surgery (Prof. Boggi, Pisa University). After this, Whipple operations were developed to a structured and standardized approach which we share in this article. The volume of $>100$ pancreatic resections per year at our hospital allowed selective choice of indications in the learning curve of the procedure.

\section{Medical Information and Informed Consent before the Operation}

Patients are informed in detail about the operation and the extension to a robot-assisted approach. The experience with the program, as well as the learning curve, is extensively discussed with the patients before the operation. This approach gives the patient the opportunity to reject it and ask for a different approach (e.g. laparoscopic). Written informed consent was received from every patient.

\section{Preparation}

The patient is normally hospitalized 1 day before the operation. The day before, the patients receive natriumpicosulfat once to reduce air, optimize visibility, and avoid disturbance by a distended intestine. Furthermore, each patient gets thrombosis prophylaxis with low-molecular-weight heparin. Approximately $30 \mathrm{~min}$ ahead of the operation every patient receives an antibiotic prophylaxis with a third-generation cephalosporine and metronidazole.

\section{Positioning of the Patient}

The patient is put in Y-position (modified beach chair position) on a vacuum mattress, and both arms are fixed close to the body to avoid damage of the brachial plexus. We use foot support and side support to ensure full stabil- 


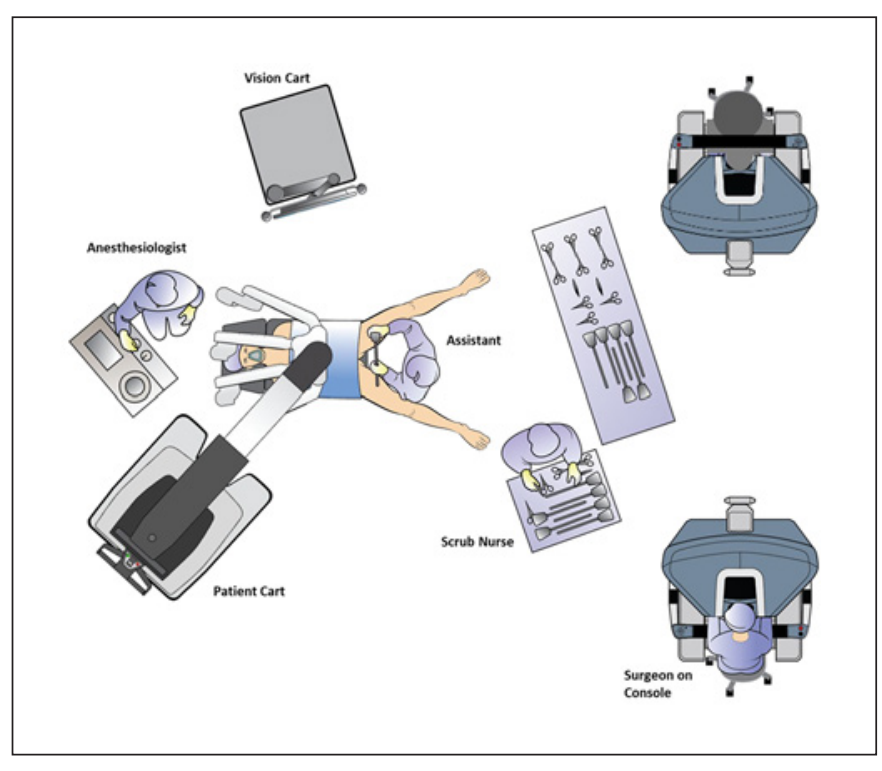

Fig. 1. Positioning of the patient (Intuitive Surgical). Before docking the trocars with the robot the operation table is moved $15^{\circ}$ reverse Trendelenburg position and canted $8^{\circ}$ to the patient's left side.

ity of the patient when the operation table is moved. All extremities are put into padding to avoid positioning nerve injury throughout the long procedure (Fig. 1).

The lower extremities are fixed to the table using elastic bandages. When using the vacuum mattress, it is important to ensure that the arms of the patient are protected on the one hand, while on the other hand the vacuum mattress has to be as flat as possible to avoid collision with the arms of the robot. The same applies for the anesthesia metal protector at the head of the patient - we routinely use 2 protectors, 1 from the left and 1 from the right, and cross them on top of the patient's face to protect it from the movement of the arms of the robot. The anesthesia metal protectors have to be installed as low and as close to the end of the operation table (head side) as possible. These measures are intended to avoid any limitations to the movement of the robot arms.

Once everything is in place we carry out a moving check to avoid problems resulting from shifting of the table or any accidental slipping of the patient. Afterwards, we are ready for disinfection and sterile covering of the operation area.

\section{Trocar Placement}

When we carry out robot-assisted pancreas procedures we usually use an additional supporting trocar to aid the 4 robotic arms. We initiate the trocar placement by putting the assist trocar in place first. We use a first entry trocar because it only requires a small incision,

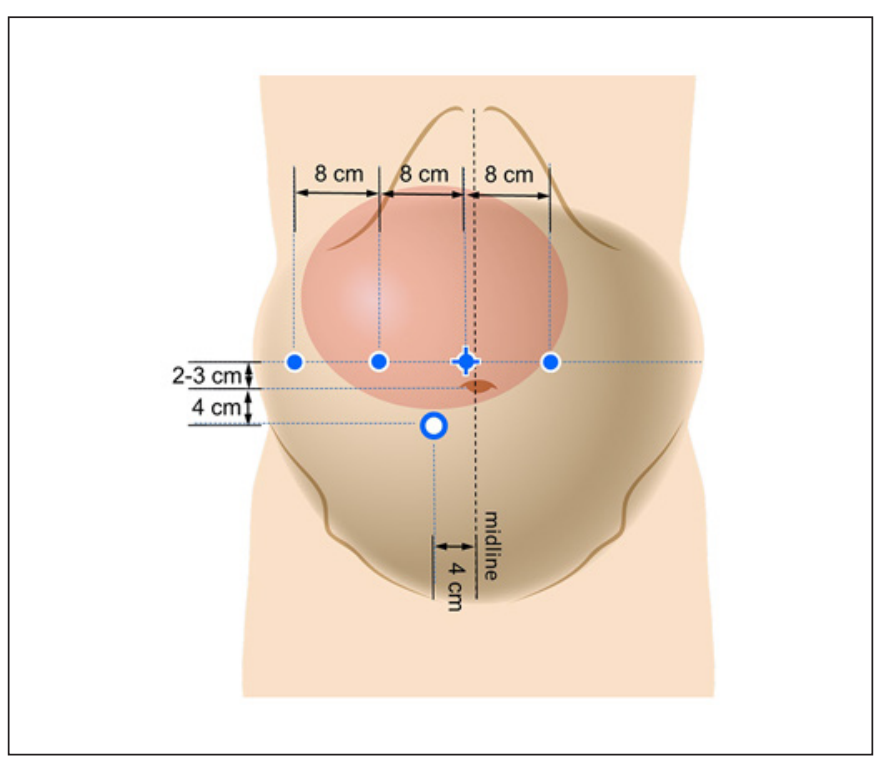

Fig. 2. Trocar placement for performing a PPPD (Intuitive Surgi$\mathrm{cal}$ ). The assist trocar is placed approximately $4 \mathrm{~cm}$ to the right side of the patient's umbilicus (radius) and $4 \mathrm{~cm}$ underneath the belly button (diagonal line). The 4 robotic trocars are placed on a straight horizontal line with a distance of $20 \mathrm{~cm}$ from the operation field and a respective distance of at least $8 \mathrm{~cm}$.

which reduces the risk of $\mathrm{CO}_{2}$-leakage or even $\mathrm{CO}_{2}$-retention of the patient (Kii Fios First Entry Access system; Applied Medical, Rache Santa Margarita, CA, USA).

\section{Pylorus-Preserving Pancreatoduodenectomy}

The size of the first entry assist trocar is $12 \mathrm{~mm}$. It is placed approximately $4 \mathrm{~cm}$ to the right of the patient's umbilicus (radius) and $4 \mathrm{~cm}$ below the belly button (diagonal line). Following the application of the pneumoperitoneum we start with a diagnostic laparoscopy using the assist trocar. This precedes the placement of 4 incision points for the robotic trocars with a distance of $20 \mathrm{~cm}$ from the operational field/ head of the pancreas, which usually sits about $2-3 \mathrm{~cm}$ above the umbilicus. We prefer the trocar placement on a straight horizontal line. The distances between the trocars are exactly measured with a ruler. The first robotic trocar is placed next to the median line on the patient's right side. Afterward, we place the other trocars with a respective distance of at least 8 $\mathrm{cm}$. As a result, after completion of the placement there should be 3 trocars in the right hemiabdomen und 1 trocar in the patient's left hemiabdomen. In the meantime, we remove any adhesions which could potentially disturb the robotic trocars (Fig. 2).

Before docking the trocars with the robot the operation table is moved $15^{\circ}$ in reverse Trendelenburg position and canted $8^{\circ}$ to the patient's left side. Table motion throughout the procedure is usually not required during the operation (Fig. 1). 


\section{Docking}

After trocar and port placement, docking of the patient cart to the trocars is performed. The patient cart of the $\mathrm{Da}$ Vinci Xi system is approached from the upper right side of the patient (Fig. 3). After connection of the camera trocar, which is the second one on the patient's right side, targeting is performed. The focus has to be set on exact placement of the remote center to avoid unnoticed dislocation which may lead to subcutaneous emphysema and $\mathrm{CO}_{2}$ retention which may require conversion or interruption of the procedure. Before commencing the procedure the position of the robotic arms is controlled and it is ensured that there is enough space (at least 1 fist) between the robot arms. After completion of the preparation the instruments are inserted under active visual observation.

We start the operation with the bipolar fenestrated forceps at the first port. The camera is in the second port, the third trocar is occupied with the bipolar scissors, and at the fourth trocar we put an instrument for passive holding purposes (small grasping retractor). During the operation we change the instruments depending on the individual situation. Quite often we use for stepwise preparation the Maryland, depending on the individual situation, especially for lymphadenectomy. For dissection of bigger vessels, except dissection of vessels near the mesentericoportal axis, we often use the vessel sealer.

\section{Standard Operating Procedure of the Procedure}

\section{Pylorus-Preserving Pancreatoduodenectomy}

Access to the Pancreas and Visualization (Step 1). Dissection of the gastrocolic ligament is performed with preservation of the gastroepiploic arcade while mainly using the vessel sealer. The stomach is lifted by the small grasping retractor (fourth trocar). The table assistant pulls the colon/major omentum downward for good exposure. Dissection of the gastrocolic ligament close to the gastroepiploic arcade ensures passive traction of the transverse colon downward in the applied reverse Trendelenburg position. To localize the tumor and to check the distance from the tumor to the mesentericoportal axis, an intraoperative ultrasound might be performed. The umbilical ligament is fixed to the upper abdominal wall via a transcutaneously placed 2-0 suture with a straight needle (Fig. 4).

Extended Kocher Manoeuvre (Step 2). Mobilization of the right colonic flexure along the duodenal rim (pars II and III) is performed while the stomach is pulled to the left upper quadrant (arm 4). Subsequently the liver is pulled up (arm 4) and a Kocher manoeuvre is performed mainly with the vessel sealer (arm 3) from part I to part III of the duodenum. In a counterclockwise preparation

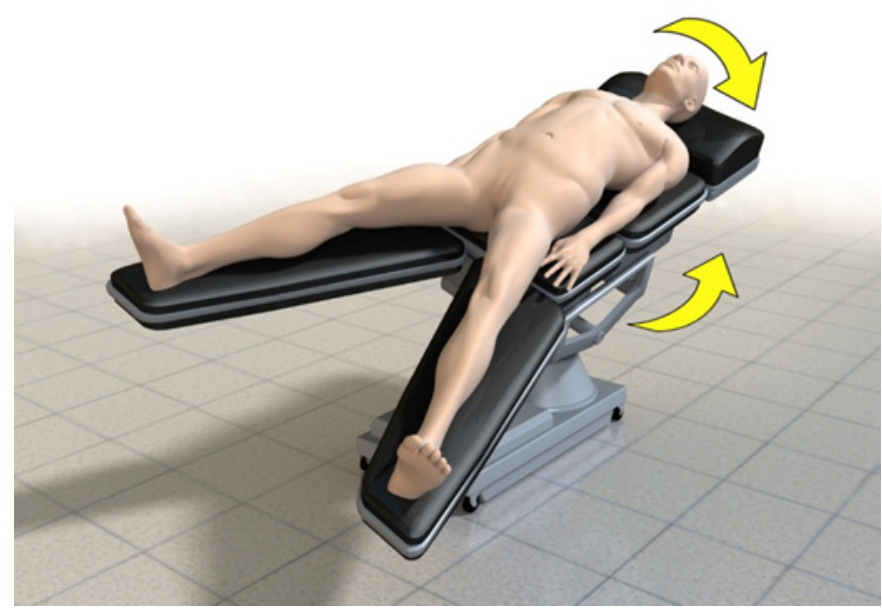

Fig. 3. Setting of a robotic operation (Intuitive Surgical). The patient cart of the Da Vinci Xi system is approached from the upper right side of the patient. The assistant is sitting between the legs of the patient. Positioning of the patient (Intuitive Surgical). Before docking the trocars with the robot the operation table is moved $15^{\circ}$ reverse Trendelenburg position and canted $8^{\circ}$ to the patient's left side.

the duodenum is mobilized in part IV and the mesentericoportal axis is prepared at the uncinate process.

Lower Rim and Mesentericoportal Axis (Step 3). Preparation and mobilization of the head of the pancreas and exposure of the vessels at the lower order of the pancreas are done to visualize the mesentericoportal axis caudally. During this step, while mainly the Maryland bipolar forceps is used alternating with scissors, the stomach and pylorus are pulled upward toward the liver. Exposure of the vessels is performed caudally to the pancreas. First we aim to expose the V. mesenterica superior. As lead compound we use the veins of the Henle loop, i.e., the V. gastroepiploica dextra and the V. colica media are prepared down to confluence with the $\mathrm{V}$. mesenterica superior and the remaining branches to the colonic flexure are dissected. Then the $\mathrm{V}$. gastroepiploica dextra is cut after clipping next to the mesentericoportal axis. After preparation of the V. mesenterica superior at the lower part of the pancreas and careful preparation along the axis underneath the pancreas, we continue uncovering a few more centimeters of the lower part of the pancreas in the direction of the pancreas cauda.

This step is followed by preparation of the A. gastroepiploica dextra. It is circularly uncovered at the head of the pancreas and ligated. Thy pylorus is circularly prepared, and then the duodenum is cut $1 \mathrm{~cm}$ postpyloric by the table assistant using an endostapler (Endo GIA, purple, $60 \mathrm{~mm}$; Medtronic, Dublin, Ireland) via an assist port. The mobilized stomach can now be placed in the upper left abdomen for the remaining dissection phase. 
Fig. 4. Individual steps of the robotic-assisted approach are presented in intraoperative pictures. Robot-assisted PPPD of a patient with a main duct intraductal papillary mucinous neoplasm (IPMN) located in the head of the pancreas. In this case, an atypical right hepatic artery (HRA) derived from the superior mesenteric artery is present (steps 4 and 5).
Step 1 and 2: Access to the pancreas and extended kocher manoeuvre
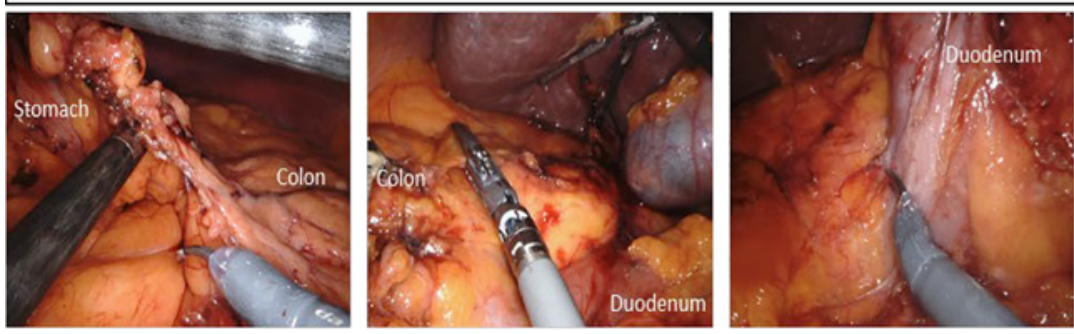

Step 4 and 5: Lower rim. mesenterico-portal axis and upper rim. hepatoduodenal
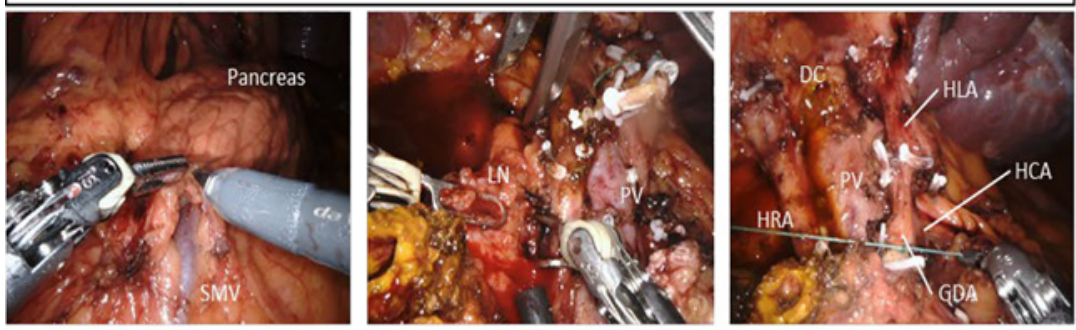

Step 5 and 6: Dissection of the pancreatic neck, mesenteric root and pars IV duodeni
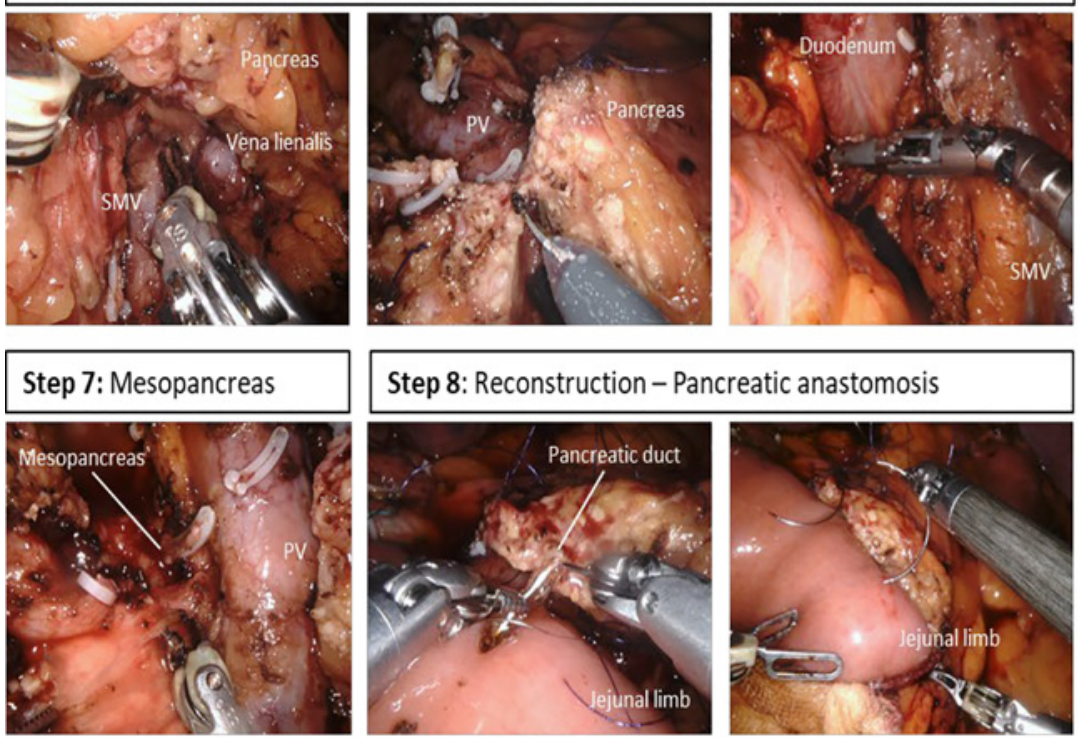

Step 8: Reconstruction - Bilio-enteric anastomosis
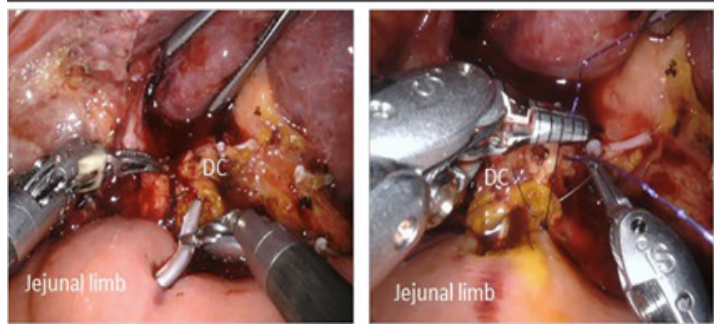

Abbreviations:

$\begin{array}{ll}\text { DC: } & \text { Ductus choledochus } \\ \text { PV: } & \text { Vena portae } \\ \text { SMV: } & \text { Superior mesenteric vein } \\ \text { GDA: } & \text { Gastroduonal artery } \\ \text { HCA: } & \text { Common hepatic artery } \\ \text { HRA: } & \text { Right hepatic artery } \\ \text { HLA: } & \text { Left hepatic artery } \\ \text { LN: } & \text { Lymph nodes }\end{array}$

DC: Ductuscholedochus

GDA: Gastroduonalartery

HCA: Commonhepatic artery

LN: Lymphnodes
Upper Rim and Hepatoduodenal Ligament (Step 4). Preparation is now continued at the start from the upper part of the pancreas. Arm 4 elevates the liver, and the table assistant softly pushes down on the pancreas (caudal).
Opening the lesser sac (omentum minus) and lymphadenectomy at the hepatoduodenal ligament with the Maryland bipolar forceps alternating with the scissors are performed. The key leading structure at this step of the pro- 
cedure is the lymph node at the upper rim of the pancreas. After removal of this lymph node the hepatic artery is visualized and lymphadenectomy is continued toward the celiac trunk. This lymph node package is then evolved towards the hepatoduodenal ligament uncovering the structures there.

Lymphadenectomy is performed on the right side and subsequently on the left side of the hepatoduodenal ligament. The A. hepatica communis, the A. hepatica propria, the Aa. hepatica dextra and sinistra and the A. gastroduodenalis are prepared. The vessels have to be perfectly uncovered to avoid missing an atypical or additional $\mathrm{A}$. hepatica dextra, which might run tangential to the DHC and origin from the superior mesenteric artery.

After preparation of the vascular structures of the hepatoduodenal ligament antegrade cholecystectomy is performed and the common bile duct is dissected right above the insertion of the cystic duct to the common bile duct. The A. gastrica dextra and the A. gastroduodenalis are separated and divided while the A. gastroduodenalis is triple ligated by 2 clips and 1 nonresorbable thread.

After dissection of the right gastric artery and the gastroduodenal artery the lympadenctomy is complete and the $\mathrm{V}$. portae is prepared up to the upper part of the pancreas.

Dissection of the Pancreatic Neck (Step 5). At this point the levels of preparation from the lower plane and the upper plane are meeting. Preparation of the portal vein by tunneling the pancreas is completed. After uncovering the V. portae at the upper part of the pancreas we continue the preparation of the $V$. portae from the lower part of the pancreas. While doing this, the pancreas is tunneled step by step until the preparation level of the caudal portion aligns with the preparation level of the cranial portion in the area of the mesentericoportal axis and the pancreas is finally locally undermined.

Once the pancreas is locally undermined at the mesentericoportal axis the pancreas parenchyma is cut through at the neck of the pancreas. We prefer cutting the pancreas step by step with monopolar scissors after coagulation with Maryland bipolar forceps. To avoid bleeding from the upper and lower arcades at the rim of the pancreas 2 stay sutures each are placed at the lower and upper rims of the pancreas. The pancreatic duct should be identified and remain slightly protuberant in relation to the head of the pancreas. No electrocautery is used at the pancreatic duct. After the pancreas is cut, the pancreas is mobilized by approximately $3 \mathrm{~cm}$ towards the cauda along the $\mathrm{V}$. lienalis (slightly pulling up the pancreatic tail on the stay sutures with arm 4). Normally there are 2 small vessels to the remaining pancreatic body and tail and arising from the A. lienalis and the A. mesenterica superior which have to be clipped.
Mesenteric Root and Pars IV Duodeni (Step 6). The next step consists of preparing the first jejunal loop mainly with the vessel sealer. This step is challenging especially in overweight patients. The preparation is continued along part IV of the duodenum from the right side of the mesenteric route and underneath the mesentericoportal axis up to the ligament of Treitz. The preparation goes far to the left side until complete mobilization of the first jejunal loop is achieved. Preparation of the mesentery of the jejunum is performed again on the right side of the mesenteric route closely along the gut until the uncinate process is completely liberated (arm IV pulls the duodenum away from the mesentericoportal axis to the right upper quadrant). The jejunum is not cut until the jejunum is fully mobilized into the upper quadrant for further reconstruction. Consecutively the jejunum then later runs via the bed of the former duodenum.

Mesopancreas (Step 7). The next step involves the preparation of the mesopancreas and uncinate process along the first venous branch of the jejunum and later along the superior mesenteric artery in cranial direction. Maryland bipolar forceps and monopolar scissors are mainly used. Branches from the superior mesenteric artery are divided with clips whenever possible. In the end this preparation level will be merged with the previous preparation level from the beginning as part of the Kocher manoeuvre (A. mesenterica first approach) close to the aorta. Sometimes it can be helpful if the table assistant carefully pushes the $\mathrm{V}$. mesenterica superior to cranial portion and left lateral with a 5-mm swab. The branches of the A. mesenterica superior, the Aa. pancreatico-duodenalis inferior and superior, are clipped. Smaller venous branches of the pancreas on the mesentericoportal axis are clipped as well. Once this is completed the dissection is finished and the specimen can be removed. The specimen is removed via a little epigastric median laparotomy as this access allows specimen removal without repositioning or undocking. For the removal a small Alexis retractor is inserted (Alexis O Wound Protector with a laparoscopic cap; Applied Medical).

Pancreatic Anastomosis Reconstruction (Step 8). In contrast to open reconstruction where we prefer pancreatogastrostomy - due to the lack of haptic feedback when inserting the pancreas to the stomach - we prefer a Blumgart style pancreaticojejunostomy when carrying out a procedure with the robot. The main reason for this is that it is unwieldy to put the cauda of the pancreas in the lanced stomach and may damage the rest of the pancreas.

When applying the Blumgart style pancreatic jejunostomy $[4,5]$ we use a black diamond microforceps ( $\operatorname{arm} 3)$ and a large needle holder (arm 1). We use 3 U-guided sutures with needles at both ends (double armed and shortened PDS 4.0 MH needle, $15 \mathrm{~cm}$; Ethicon, J\&J, USA). These are used for the $3 \mathrm{U}$-shaped transpancreatic sutures 
through the full width of the pancreas, which create overlapping of the jejunum at the upper and lower parts of the anastomosis. After prepositioning the sutures at the lower pancreatic parenchyma a duct-to-mucosa anastomosis is performed with stenting (Pflugbeil pancreas stent PancreaPlus 6 F; Pflugbeil, Zorneding, Germany) of the pancreatic duct (5-0 PDS C1 needle, $10 \mathrm{~cm}$; Ethicon, J\&J) and the duct-to-mucosa anastomosis are encapsulated by completion of the anterior aspect of the anastomosis with the prepositioned 4-0 MH needles. The duct-to-mucosa anastomosis is performed in a circular fashion by interrupted sutures after making a little orifice for the pancreas duct in the jejunum.

Bilioenteric Anastomosis Reconstruction (Step 9). After finishing the pancreatojejunostomy we turn to the biliodigestive anastomosis. We again use a black diamond microforceps ( $\operatorname{arm} 3$ ) und a large needle holder (arm 1). The approach is analogous to the open method with single sutures (PDS $5.0 \mathrm{C} 1$ needle, $10 \mathrm{~cm}$; Ethicon, J\&J). After incising a little orifice in the jejunum with the monopolar robotic scissors we suture the posterior wall using single interrupted sutures. To reduce tension on the anastomosis, fixation of the jejunal loop to the liver might be necessary with a single 4-0 monofilament suture (4-0 PDS SH needle, Ethicon, J\&J, USA). This fixation is usually performed in the bed of the gallbladder. Before finishing the anterior wall, we place a "lost" stent to support the biliodigestive anastomosis (see above).

Dudenojejunal Anastomosis Reconstruction (Step 10). The duodenojejunal anastomosis might be challenging in the sense that mobilization of the inframesocolic small bowel by robotic instruments might be complicated due to the angle of the inserted robotic arms. Reconstruction can therefore alternatively easily be performed via the retrieval incision which has been closed with a laparoscopic cap after specimen retrieval. Reconstruction is performed as an end-to-side-anastomosis to a jejunal omegaloop (running suture PDS $4.0 \mathrm{SH}, 15-\mathrm{cm}$ needle; Ethicon, J\&J) using a large needle holder (arm 3). We leave the ends of the suture long, and thus it is possible to lift the anastomosis with a holding instrument using the robot (arm 4). The other side may be held by the table assistant. Then the clips of the endostapler are removed while opening the duodenum. The anastomosis is finished with a single continual running suture at the anterior wall of the anastomosis.

Drainage and Closure (Step 11). Before the operation is finished, 4 Easy Flow drains are placed, i.e., 2 in front and behind the biliodigestive anastomosis and 2 behind and in front of the pancreatic anastomosis. In robot-assisted pancreatic surgery routine drains are used. The trocars are removed under active visual observation to detect any bleeding and the wounds are closed (PDS 2.0 UR 6 suture; Ethicon).

\section{Discussion}

In recent years surgical technology, like robot-assisted procedures, has developed very much and is increasingly used in complex visceral surgical procedures. Even though the benefit of this technology in pancreatic surgery has not been proven, feasibility and safety studies have resulted in a much wider acceptance of this technology than of laparoscopic techniques in the last decade.

There are subjective benefits for the surgeon, like better handling and angulation, ideal visualization, and less physical exertion, that hold potential for a wider acceptance of the robot in minimal invasive pancreatic surgery than laparoscopic techniques. However, given the above mentioned benefits, i.e., in terms of operative time, blood loss, morbidity rate, $\mathrm{POPF}$ rate, rate of reoperation and mortality, has to be considered. It is a matter of concern that there is a flat learning curve for the surgeon, which might be due to the summation of a lack of forced feedback, the complexity of the procedure, and the circumstances, structured program, and case load available for training.

A retrospective study of Shi et al. [6] which includes 450 patients who underwent a robot-assisted pyloruspreserving pancreatoduodenectomy showed that blood loss, operational time, and pancreatic leak depends on the frequency with which the procedure is done, otherwise also described as the learning curve of the surgeon. The more often a surgeon did the procedure the more the operative time improved. While procedures 1-50 took 405.4 $\pm 112.9 \mathrm{~min}$, procedures $301-350$ took $273.6 \pm 70 \mathrm{~min}$ $(p<0.001)$. The same applies to blood loss. While the estimated blood loss amounted to $410 \pm 563.5 \mathrm{ml}$ in cases $1-50$, it could be reduced to $149 \pm 103.3 \mathrm{~mL}$ in cases $351-$ $400(p<0.001)$. Furthermore, the incidence of pancreatic leak decreases significantly in the last 350 procedures against cases $1-100$ (30.0 vs. $15.1 \%, p=0.003$ ). The conclusion of Shi et al. [6] is that the operative outcome, as well as the oncologic outcome, clearly improves if more than 250 procedures are performed.

We argue that a structured training program and simulation as well as wet lab training can significantly reduce this number. Other data in this context show that the learning curve can be optimized after about 40 [7] or 80 cases $[2,8]$.

The key step to improving the learning curve, in our opinion, is structured and dedicated training programs. We could previously demonstrate that structured video tutorials can improve surgical skills in the OR even if not in a procedure-specific manner [9]. To practice a procedure, training programs and standardization of the operation into key steps can be performed. Structured training programs have been developed to learn procedures with the Da Vinci system stepwise. The University of Pittsburgh Medical Center established a stepwise training 
program for pancreatic resections to disseminate safe robot-assisted pancreatectomy. Their program includes the following 5 main components: a curriculum with virtual reality simulation, a simulation curriculum with inanimate biotissue, video library drills, intraoperative coaching, and continuous assessment and quality improvement $[2,3]$. A stepwise curriculum to shorten the learning curve should be expanded to relevant centers worldwide.

There are different opinions on how to perform the pancreatic anastomosis after pancreatoduodenectomy in a robot-assisted fashion. The most feared and relevant complication after PPPD is a postoperative pancreatic fistula (POPF). A retrospective review at University of Illinois Hospital and Health Sciences System investigated the risk factors and the incidence of POPF after different types of pancreatic anastomosis with a robot-assisted approach. Included were 69 patients who underwent a robot-assisted pancreatoduodenectomy. In that study the only significant risk factor for POPF was a pancreatic duct diameter $<3$ $\mathrm{mm}$. The study showed that reconstruction with pancreaticojejunostomy and transgastric pancreatogastrostomy has a lower risk of POPF than pancreatic duct occlusion with cyanoacrylate glue and posterior pancreatogastrostomy [10]. The role of pancreatogastrostomy versus pancreaticojejunostomy as far as the safety of the anastomosis is concerned remains disputed [11-13]. Even though we are promoting pancreatogastrostomy [14-16] in open and laparoscopic operations the drawback of this reconstruction in the robot-assisted setting might be that it leads to traumatization of the organ during the process of invagination into the stomach for the purse string suture by the lack of forced feedback. We therefore stick to the reconstruction where established models enable training in a biotissue setting [17]. More prospective randomized studies are needed to get definitive results on the value of the different reconstructive techniques in a robot-assisted setting. In conclusion, there are some studies which show that robotic-assisted pancreaticojejunostomy is safe and feasible $[18,19]$.
In the review article of Liu et al. [20], which evaluated the current status of robot-assisted pancreatic surgery, it is documented that robot-assisted PPPD has a perioperative mortality rate similar to that of the open approach, and the postoperative rates of complications and the POPF rates are comparable, while the robot-assisted method has a shorter hospital stay (moderate level of evidence) [20]. Furthermore, in the same review it is documented that robot-assisted pancreatoduodenectomy was associated with a higher rate of $\mathrm{R} 0$ resection of malignant tumors compared to the open method, while the number of harvested lymph nodes was similar (moderate level of evidence) [20]. In summary, more studies are needed to get definitive results.

\section{Statement of Ethics}

All images were obtained with informed consent from the patient. An ethics committee statement does not apply.

\section{Disclosure Statement}

There are no relevant disclosures from any of the authors.

\section{Funding Sources}

This work was only financed by departmental funds.

\section{Author Contributions}

C.F. Müller-Debus: drafting of this paper and literature research. M. Thomaschewski and M. Zimmermann: images and video analysis. U.F. Wellner and T. Keck: operations and correction of the draft of this paper. D. Bausch: correction of draft of this paper.

\section{References}

1 Siech M, Strauss P, Huschitt S, Bartsch DK, Wittel U, Keck T. The Indications for Laparoscopic Pancreatectomy. Dtsch Arztebl Int. 2017 Apr;114(15):263-8.

2 Kim A C, Rist R C, Zureikat A H Technical Detail for Robot Assisted Pancreaticoduodenectomy. J Vis Exp. 2019 Sep 28;(151).

3 Mark Knab L, Zenati MS, Khodakov A, Rice M, Al-Abbas A, Bartlett DL, et al. Evolution of a novel robotic training curriculum in a complex general surgical oncology fellowship. Ann Surg Oncol. 2018 Nov;25(12): 3445-52.

4 Grobmyer SR, Kooby D, Blumgart LH, Hochwald SN. Novel pancreaticojejunostomy with a low rate of anastomotic failure-related complications. J Am Coll Surg. 2010 Jan;210(1):54-9.
5 Fujii T, Sugimoto H, Yamada S, Kanda M, Suenaga M, Takami $H$, et al. Modified Blumgart anastomosis for pancreaticojejunostomy: technical improvement in matched historical control study. J Gastrointest Surg. 2014 Jun;18(6):1108-15.

6 Shi Y, Wang W, Qiu W, Zhao S, Wang J, Weng $Y$, et al. Learning curve from 450 cases of robot-assisted pancreaticoduocectomy in a high-volume pancreatic center: optimization of operative procedure and a retrospective study. Ann Surg. 2019 Oct: 1.

7 Zhang T, Zhao ZM, Gao YX, Lau WY, Liu R. The learning curve for a surgeon in robot-assisted laparoscopic pancreaticoduodenectomy: a retrospective study in a high-volume pancreatic center. Surg Endosc. 2019 Sep;33(9):2927-33.
8 Boone BA, Zenati M, Hogg ME, Steve J, Moser AJ, Bartlett DL, et al. Assessment of quality outcomes for robotic pancreaticoduodenectomy: identification of the learning curve. JAMA Surg. 2015 May;150(5):416-22.

9 Thomaschewski M, Esnaashari H, Höfer A, Renner L, Benecke C, Zimmermann M, et al. Video tutorials increase precision in minimally invasive surgery training: a prospective randomised trial andfFollow-up study. Zentralbl Chir. 2019 Apr;144(2):153-62.

10 Gonzalez-Heredia R, Durgam S, Masrur M, Gonzalez-Ciccarelli LF, Gangemi A, Bianco FM, et al. Comparison of Different Techniques of Pancreatic Stump Management in Robot-Assisted Pancreaticoduodenectomy. Gastrointest Tumors. 2019 Feb;5(3-4):68-76. 
11 Giulianotti PC, Gonzalez-Heredia R, Esposito S, et al. Trans-gastric pancreaticogastrostomy reconstruction after pyloruspreserving robotic Whipple: a proposal for a standardized technique. Surg Endosc. 2018 Apr;32(4):2169-74.

12 Aselmann H, Egberts JH, Hinz S, Jünemann KP, Becker T. Robot-assisted pylorus-preserving partial pancreaticoduodenectomy (Kausch-Whipple procedure). Zentralbl Chir. 2016 Apr;141(2):139-41.

13 Memeo R, Tzedakis S, de Blasi V, Dehlawi A, Untereiner X, Rodriguez $\mathrm{M}$, et al. Robotic Pancreaticoduodenectomy: Operative Steps (With Video). Surg Laparosc Endosc Percutan Tech. 2016 Oct;26(5):e91-4.
14 Keck T, Wellner UF, Bahra M, Klein F, Sick $\mathrm{O}$, Niedergethmann $\mathrm{M}$, et al. Pancreatogastrostomy versus pancreatojejunostomy for RECOnstruction after PANCreatoduodenectomy (RECOPANC, DRKS 00000767): perioperative and long-term results of a multicenter randomized controlled trial. Ann Surg. 2016 Mar;263(3):440-9.

15 Grützmann R, Distler M, Keck T, Niedergethmann M. Die Pankreasanastomose: ein Videovergleich unterschiedlicher Anastomosentechniken. Zentralbl Chir. 2013 Feb; 138(1):24-6.

16 Keck T, Kuesters S, Wellner U, Hopt UT, Karcz WK. Laparoscopic pylorus-preserving pancreatic head resection and hybrid open reconstruction via pancreatogastrostomy. J Gastrointest Surg. 2011 Feb;15(2):373-7.
17 Napoli N, Kauffmann EF, Menonna F, Perrone VG, Brozzetti S, Boggi U. Indications, technique, and results of robotic pancreatoduodenectomy. Updates Surg. 2016 Sep;68(3):295-305.

18 Zhao W, Liu C, Li S, Geng D, Feng Y, Sun M. Safety and efficacy for robot-assisted versus open pancreaticoduodenectomy and distal pancreatectomy: A systematic review and metaanalysis. Surg Oncol. 2018 Sep;27(3):468-78.

19 Watkins AA, Kent TS, Gooding WE, et al. Multicenter outcomes of robotic reconstruction during the early learning curve for minimally-invasive pancreaticoduodenectomy. HPB (Oxford). 2018 Feb;20(2):155-65.

20 Liu R, Wakabayashi G, Palanivelu C, Tsung $\mathrm{A}$, et al. International consensus statement on robotic pancreatic surgery. Hepatobiliary Surg Nutr. 2019 Aug;8(4):345-60. 